\title{
SAR Image Segmentation using Statistical Techniques
}

\author{
Jharna Majumdar \\ Former Sc G (DRDO) \\ Dean, R \& D, Prof. Comp Sc \& Engg. \\ Nitte Meenakshi Institute of Technology \\ Yelahanka, Bangalore - 560064
}

\author{
Lekshmi.S \\ Scientist \\ Aeronautical Development Estt. \\ C.V. Raman Nagar \\ Bangalore - 560093
}

\begin{abstract}
Segmentation is performed in recognition applications as a primary step towards extraction of interesting regions of an image. In this paper, the characteristic effects of Weibull and Fractal parameters in the segmentation of Synthetic Aperture Radar (SAR) and Optical images acquired from satellite platform is studied. The algorithms are tested for different window sizes and different number of classes to bring out the effect of these parameters in the segmentation process.
\end{abstract}

Keywords: Segmentation, Weibull

\section{INTRODUCTION}

Segmentation process partitions an image into regions that possess consistent properties. The approach to image segmentation varies depending on the characteristics of the image. The conventional approach of segmentation would classify an optical image to a considerable extent but fails to produce a reasonable result for SAR imagery. SAR images are corrupted with speckle noise and hence any standard image processing software would not produce expected result. The segmentation of Satellite SAR and Satellite optical images using Weibull and Fractal parameters is described in this paper. Furthermore, the effect of window size and cluster count on the end result is also analyzed.

\section{METHODOLOGY}

The characteristic effects of Weibull and Fractal parameters are exploited for the segmentation of SAR and optical images. The Weibull distribution is a well established theory and the statistical parameters of a Weibull distribution are commonly evaluated for life data analysis of brittle materials. In the present context, Weibull distribution is used to model a SAR image. A SAR image pixel $\left|C_{m, n}\right|$, is assumed to be a Weibull distributed variable with form parameter $\gamma$ and scale parameter $\beta$. To compute $\gamma$ and $\beta$, a window [.] of size nxn is considered that surrounds the pixel of an image. The probability density function of Weibull distribution is given by

$$
\begin{aligned}
& \mathrm{p}\left(\left|\mathrm{C}_{\mathrm{m}, \mathrm{n}}\right|\right)= \\
& \frac{\gamma_{m, n}}{\beta_{m, n}}\left[\frac{\left|C_{m, n}\right|}{\beta_{m, n}}\right]^{\gamma_{m, n}-1} \exp \left[-\frac{\left|C_{m, n}\right|}{\beta_{m, n}}\right]^{\gamma_{m, n}}
\end{aligned}
$$

where

$\gamma_{\mathrm{m}, \mathrm{n}}=\prod / \mathrm{std}\left[\ln \left(\left|\mathrm{C}_{\mathrm{m}, \mathrm{n}}\right|\right)\right] \sqrt{ } 6$

and $\beta_{\mathrm{m}, \mathrm{n}}=\exp \left\{\operatorname{mean}[\ln (|\mathrm{Cm}, \mathrm{n}|)]+0.5772 / \gamma_{\mathrm{m}, \mathrm{n}}\right\}$ std [.] and mean [.] are respectively the standard deviation and the mean of the window [.]. In this paper, the scale parameter, is considered for image segmentation [1,2].

Another parameter considered is the Fractal parameter, namely the Fractal dimension. Fractal dimension measures the degree of self-similarity of an image. In the present work, Fractal dimension is computed by variation method. Fractal dimension is computed for an image pixel by considering a window that surrounds the pixel. Variation method defines Fractal dimension as the slope of the line that best fits the points $\left(\log (\mathrm{R} / \varepsilon), \log \left\{(\mathrm{R} / \varepsilon)^{3} \mathrm{E}_{\varepsilon}\right\}\right)$ where $\mathrm{R}$ is the window size and $\varepsilon$ ranges from 1 to $\varepsilon_{\max }, \mathrm{E}_{\varepsilon}$ is taken as the average of $\mathrm{V}_{\varepsilon}(\mathrm{x}, \mathrm{y})$, where $\mathrm{V}_{\varepsilon}(\mathrm{x}, \mathrm{y})$ is the $\varepsilon^{\text {th }}$ variation (difference between maximum and minimum pixel value) over a window of size $2 \varepsilon+1[2,3,4]$. The range of Weibull and Fractal parameters varies for optical and SAR images. The Fractal parameter for both these images varies over a very narrow range. Hence it is not effective to apply these values directly for segmentation. Instead, the Weibull and Fractal parameters are mapped in the range $[0,255]$ to generate an intensity image. The histogram is constructed for the intensity image and the range of values which falls between lower and higher frequency elements with a certain threshold frequency is considered for segmentation. The range for each region is decided by the number of classes entered by the user. The range of the region $\left[a_{i}, a_{i+1}\right]$ is computed as $a_{i}=a+i * t \quad$ for $i=0,1, \ldots, c-2$, where

$\mathrm{c}$ is the user input and $\mathrm{t}=(\mathrm{b}-\mathrm{a}) / \mathrm{c}$, where $\mathrm{a}$ and $\mathrm{b}$ correspond the minimum and maximum of the selected region. The intensity values belonging to the tails of the histogram is assigned the same region that its immediate neighbor holds. The features are also segmented using clustering technique to identify the homogeneous regions in the image. After segmentation the regions are assigned pseudo colors for visual clarity.

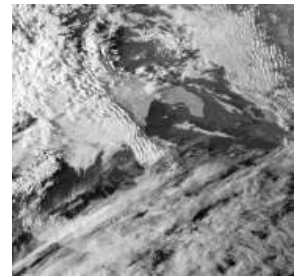

Fig 1. Optical image

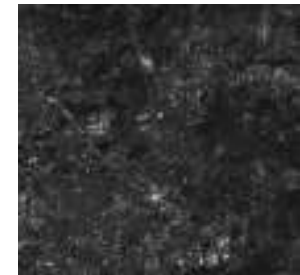

Fig 2. SAR image 


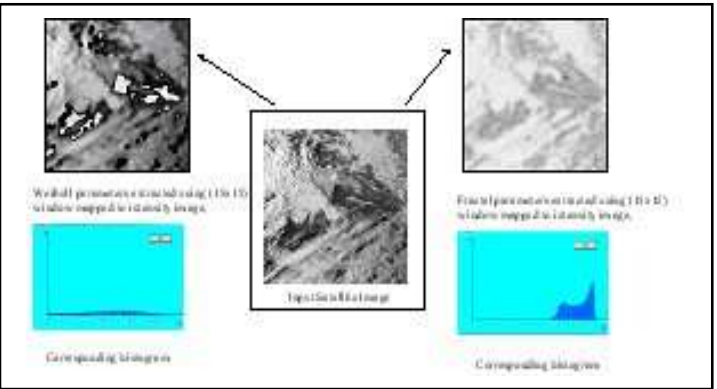

Fig 3 (a) Intensity images using Weibull and Fractal parameters (window size $=3 \times 3$ ) and the corresponding histograms for Optical image

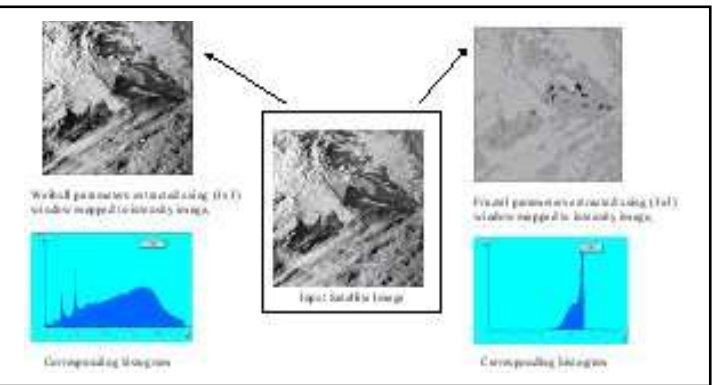

Fig 3 (b) Intensity images using Weibull and Fractal parameters (window size $=15 \times 15$ ) and the corresponding histograms for Optical image

\section{TEST RESULT}

Segmentation algorithms described above were tested using a sample optical and SAR image shown in Figs 1 and 2 respectively. The Weibull and Fractal parameters are computed for these images for a smaller window size $(3 \times 3)$ and a larger window size $(15 \times 15)$ and the corresponding intensity images are generated. An example of intensity image and its histogram computed using Weibull and Fractal parameters for both optical and SAR image are shown in Figs 3 and 4 . Figs 5 and 6 show the plot of min-max range of these parameters.

It could be observed that the segmented output image varies in quality with the change in window size used for the computation of Weibull and Fractal parameters. Hence the results were presented with varying window size. The smaller window sizes considered in the present study are $3 \times 3$, $5 \times 5,7 \times 7,9 \times 9$ and the larger window sizes considered are

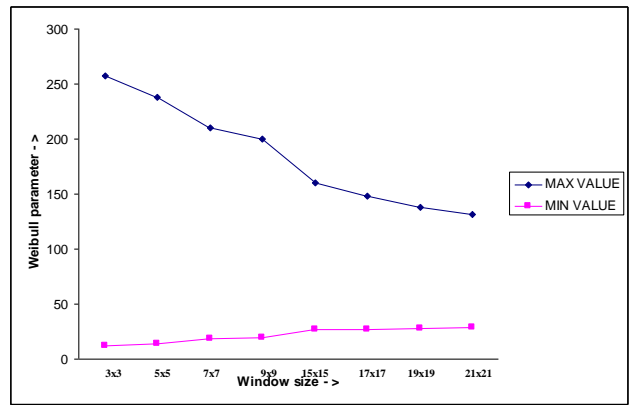

Fig 5 (a) Min and Max range for Weibull parameter computed for SAR image
$15 \times 15,17 \times 17,19 \times 19,21 \times 21$. The output images obtained with varying window size and for different number of classes for a sample optical and SAR images are given in Fig 7 and Fig 8. It is seen that Weibull parameter computed using smaller window size produced good result, whereas Fractal parameter computed using larger window size produced acceptable results. This was found to be true for both Satellite and SAR images.

The input images are also segmented using k-means clustering technique to produce segmented outputs. It could be observed that Weibull parameter computed using smaller window size is more effective in detecting local neighborhood information. In the Figs 9 to 12, it could be seen that the fine details could be detected using Weibull parameter whereas fractal parameter (Fig 9c and 10c) could segment image into coarse segments.

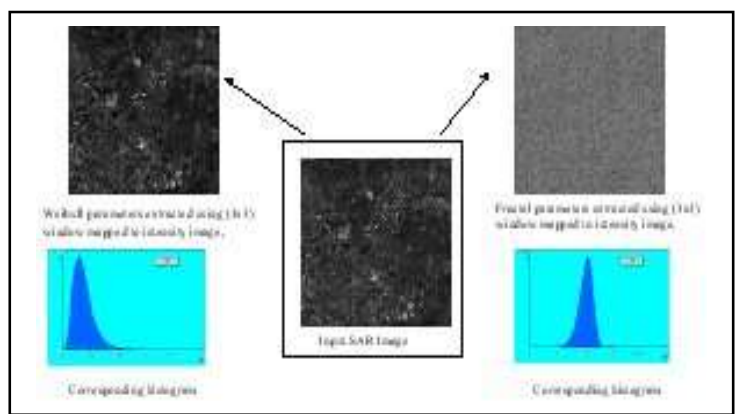

Fig 4 (a) Intensity images using Weibull and Fractal parameters (window size $=3 \times 3$ ) and the corresponding histograms for SAR image

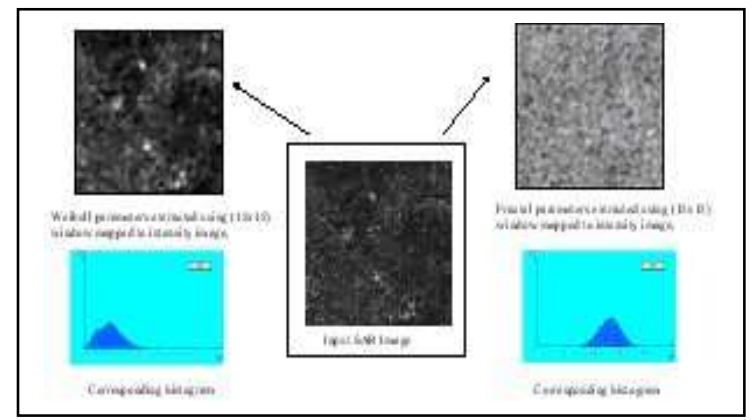

Fig 4 (b) Intensity images using Weibull and Fractal parameters (window size $=15 \times 15$ ) and the corresponding histograms for SAR image

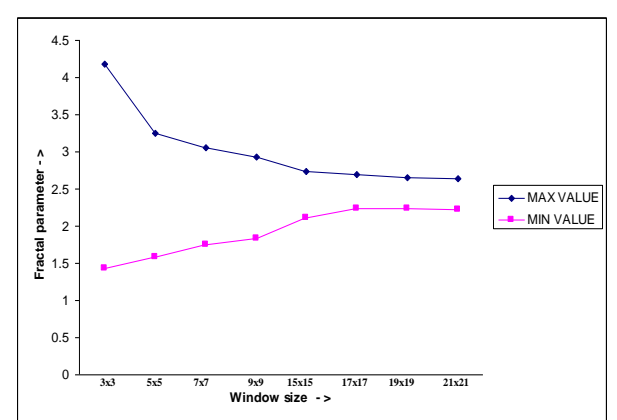

Fig 5 (b) Min and Max range for Fractal parameter computed for SAR image 


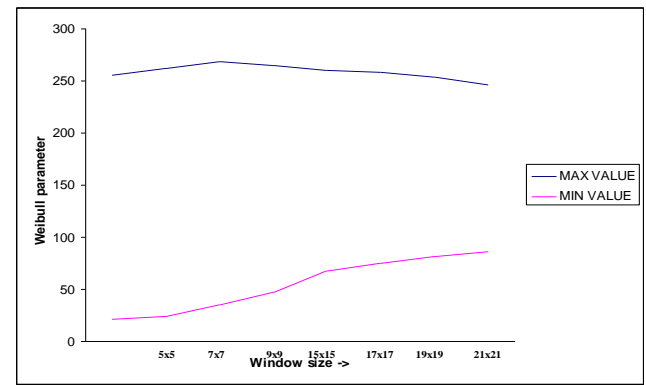

Fig 6 (a) Min and Max range for Weibull parameter computed for Optical image

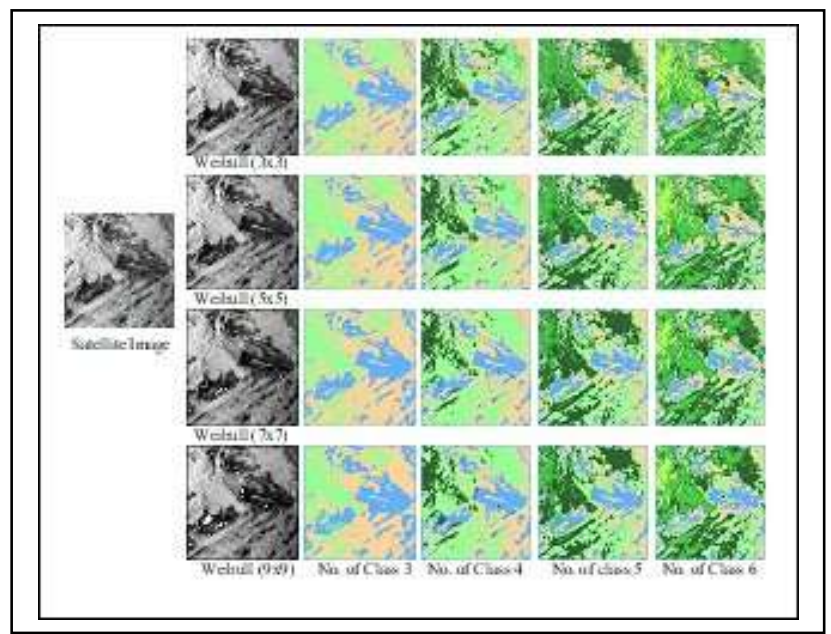

Fig 7 (a) Satellite image - Weibull output for small window sizes

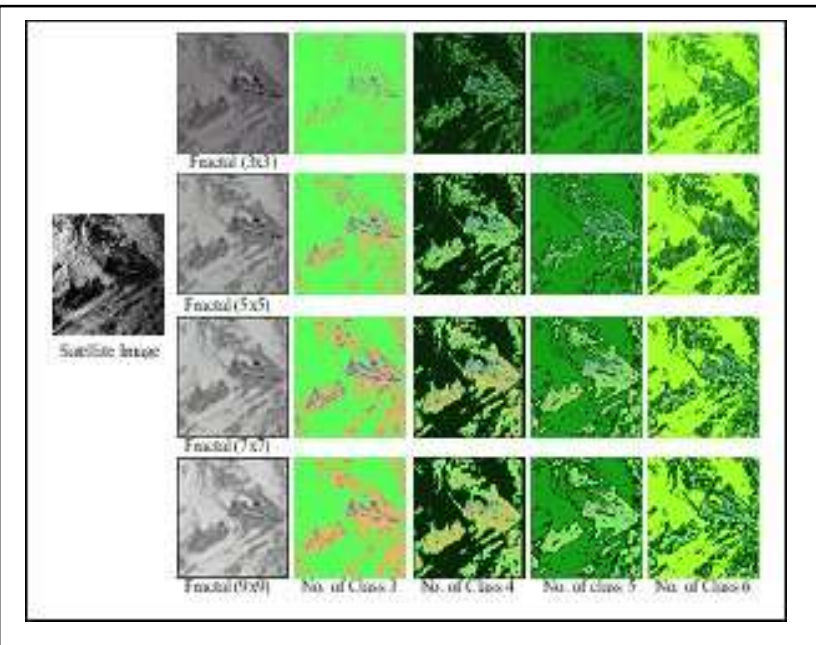

Fig 7 (c) Satellite image - Fractal output for small window sizes

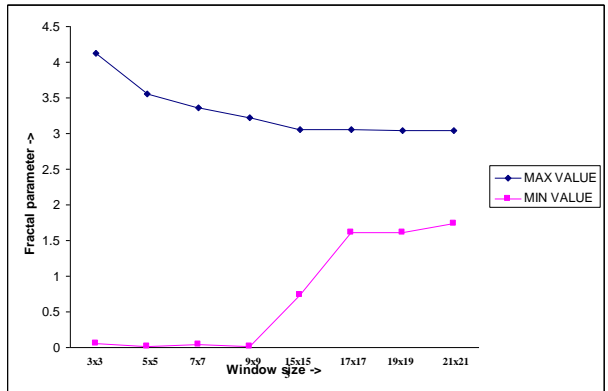

Fig 6 (b) Min and Max range for Weibull parameter computed for Optical image

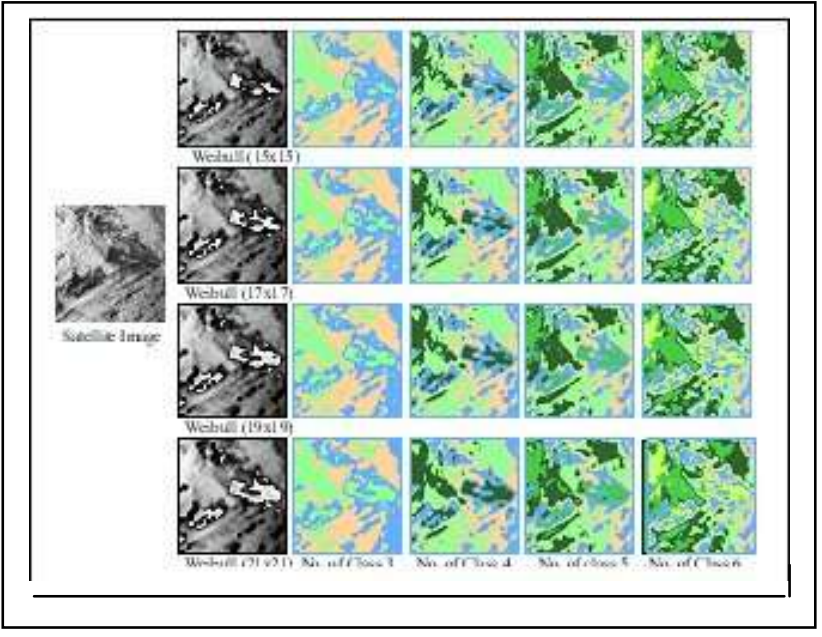

Fig 7 (b) Satellite image - Weibull output for large window sizes

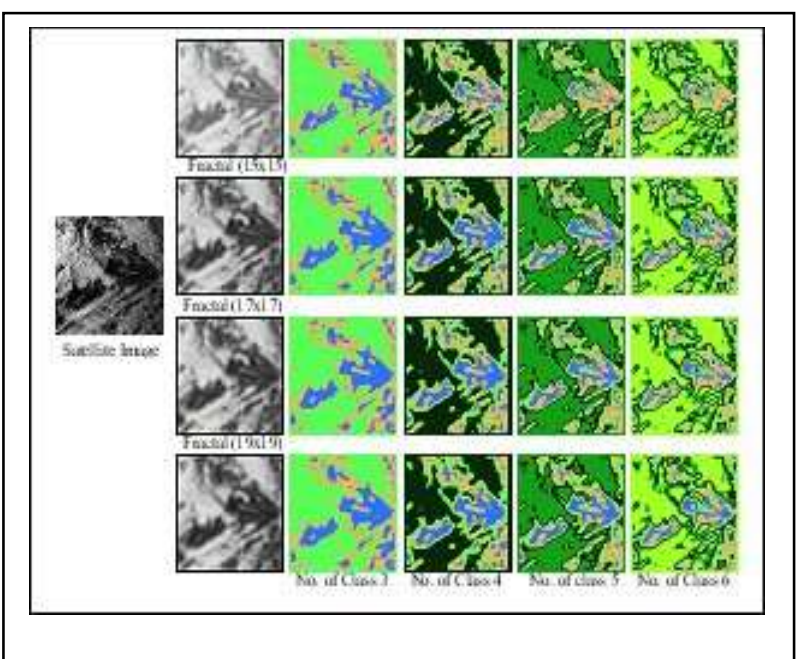

Fig 7(d) Satellite image - Fractal output for large window sizes 


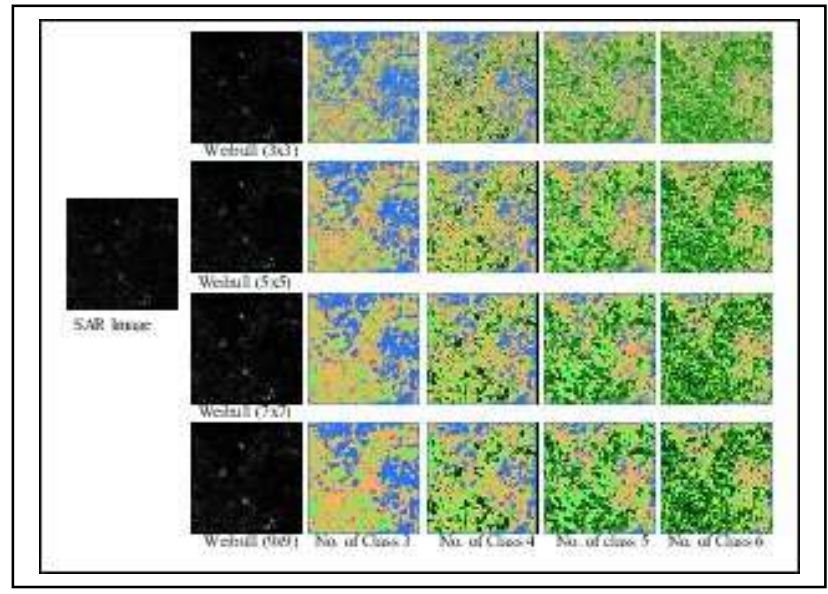

Fig 8(a) SAR image - Weibull output for small window sizes

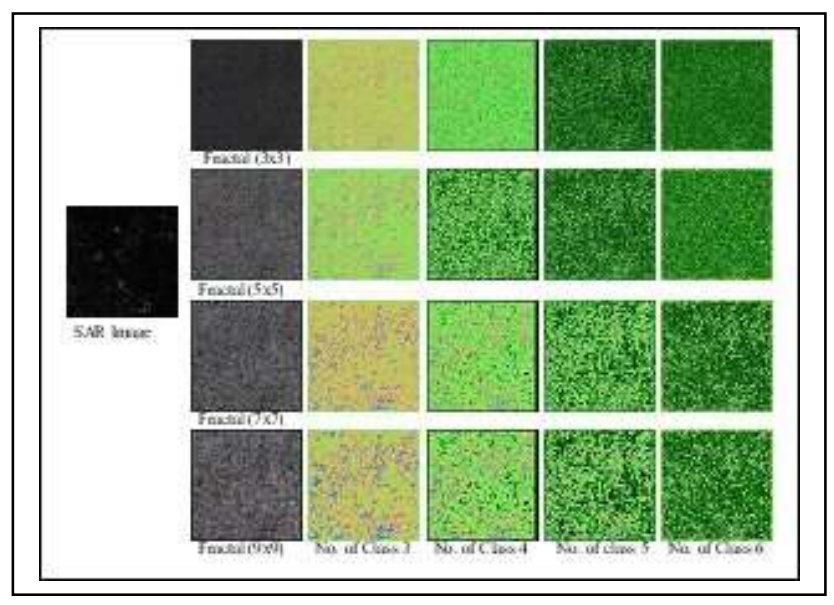

Fig 8 (c) SAR image - Fractal output for small window sizes

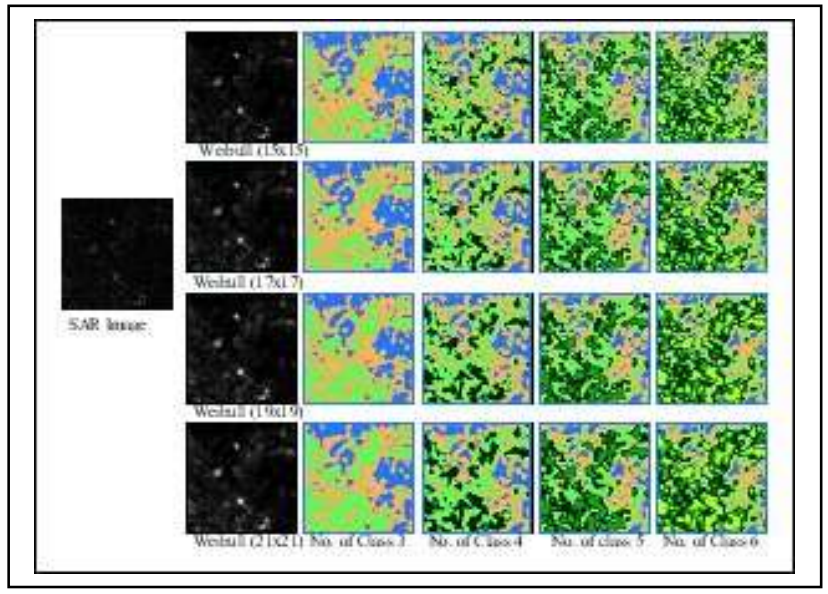

Fig 8(b) SAR image - Weibull output for large window sizes

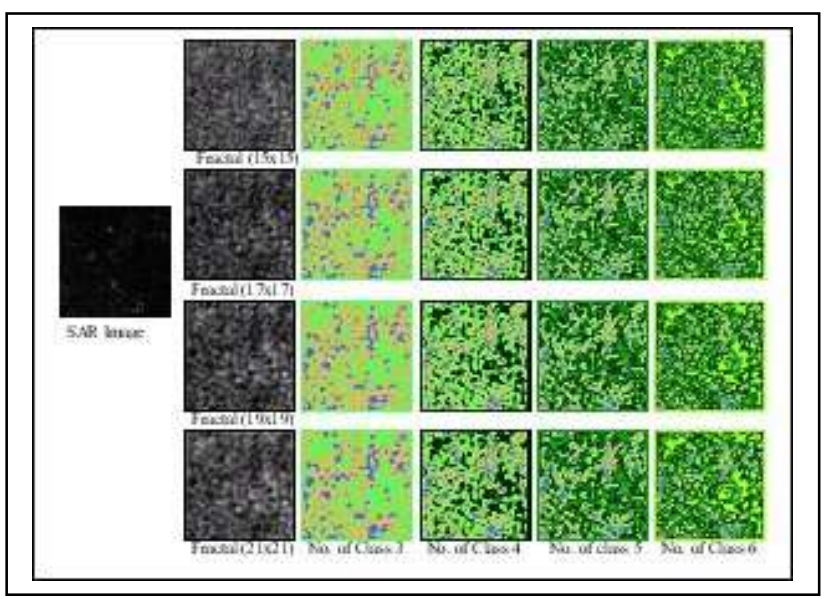

Fig 8(d) SAR image - Fractal output for large window sizes

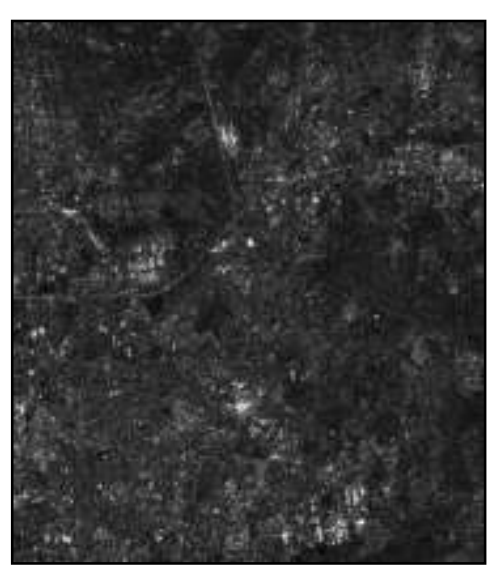

Fig 9 (a) SAR image1

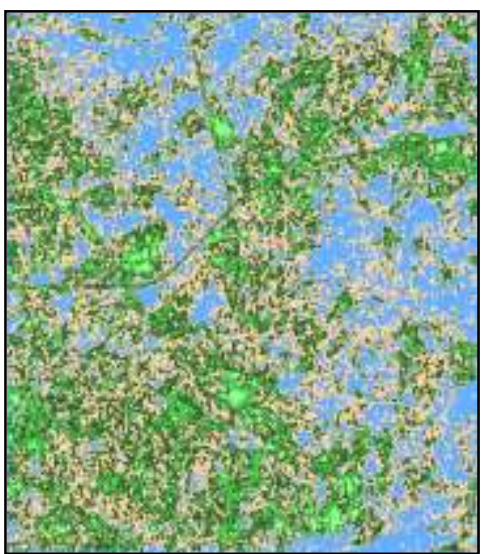

Fig 9 (b) Segmented image using Weibull parameter

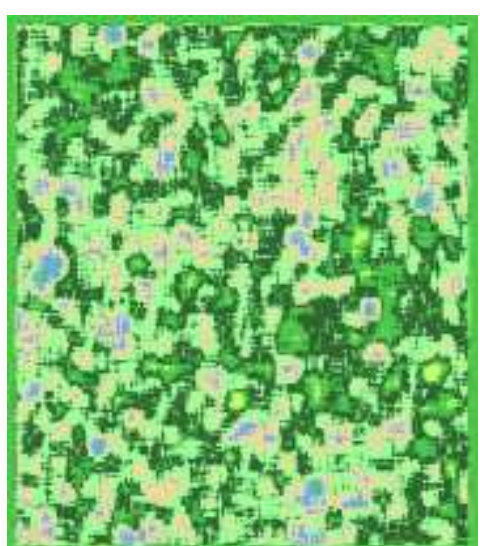

Fig 9 (c) Segmented image using Fractal parameter 


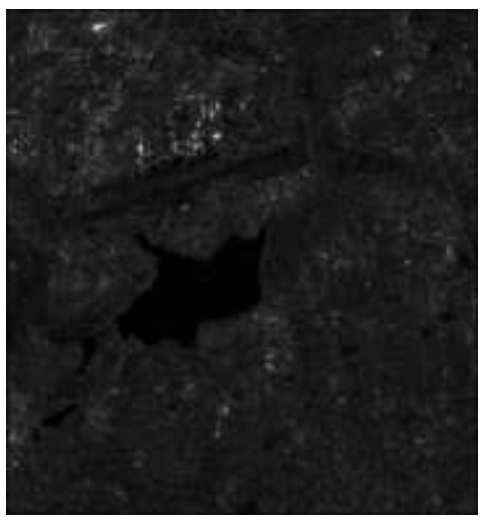

Fig 10 (a) SAR image2

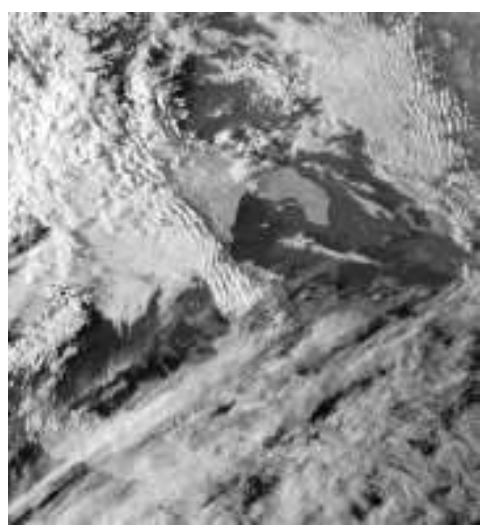

Fig 11 (a) Satellite image 1

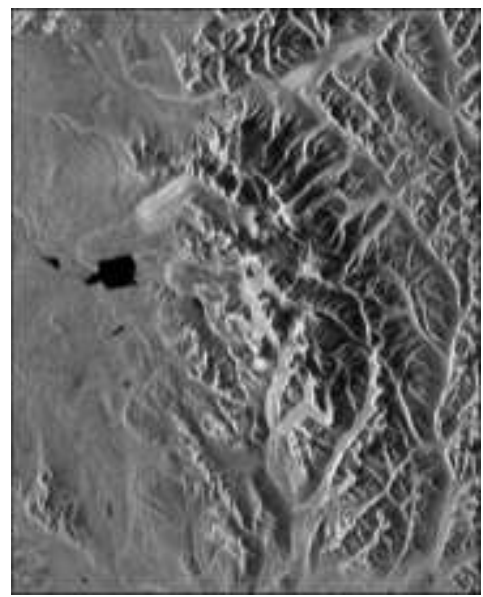

Fig 12(a) Satellite image2

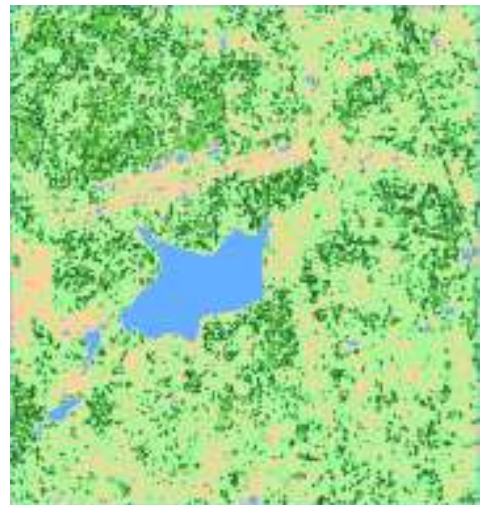

Fig 10 (b) Segmented image using Weibull parameter

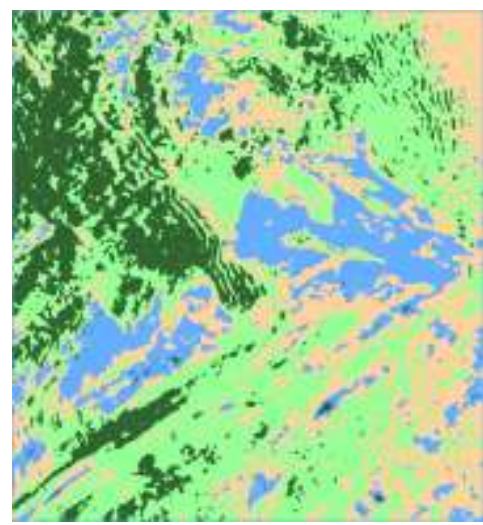

Fig 11 (b) Segmented image using Weibull parameter

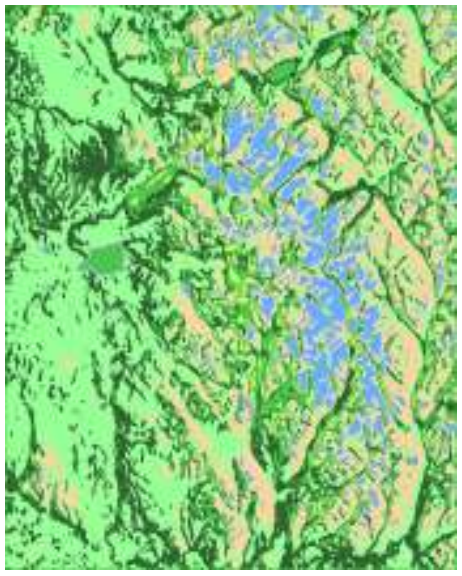

Fig 12(b) Segmented image using Weibull parameter

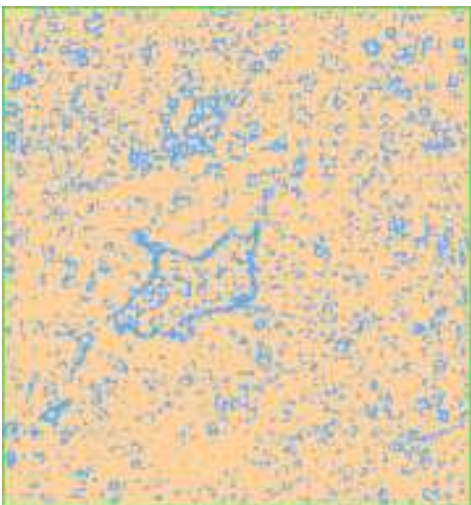

Fig 10 (c) Segmented image using Fractal parameter

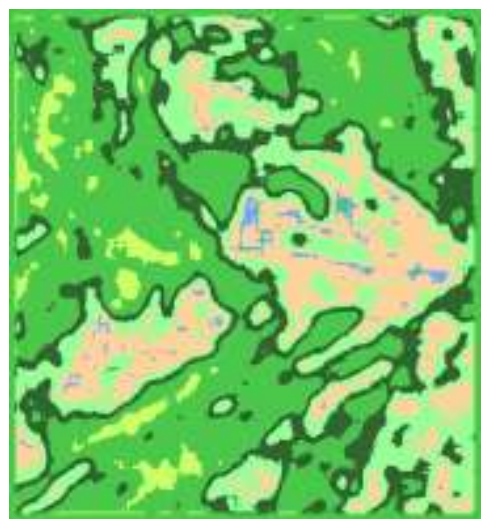

Fig 11 (c) Segmented image using Fractal parameter

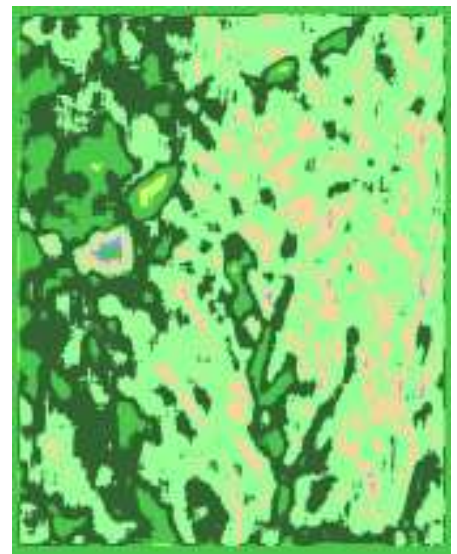

Fig 12 (c) Segmented image using Fractal parameter 


\section{CONCLUSION}

The segmentation of SAR and optical images using Weibull and Fractal parameters with varying window size and different number of classes is studied in this paper. The segmentation results were found to be good for optical images and SAR images with Weibull parameter based segmentation and smaller window size. For the Fractal parameter based segmentation, the results were good with larger window size. The study also shows that segmented output varies with the number of classes. The features were segmented using clustering method to identify regions with similar characteristics.

\section{ACKNOWLEDGEMENT}

The authors thank Mr P.S. Krishnan, Director ADE for his support and kind permission to publish the paper.

\section{REFERENCES}

[1] David Fernandes, Segmentation of SAR Images with Weibull Distribution, Int. Geoscience and Remote Sensing Symposium (IGARSS), Seatle, 1998, p.14561458.

[2] Chaudhuri, B.B. and Sarkar, N., 'Texture Segmentation using Fractal dimension', IEEE Trans on PAMI , 17 : 72-77, 1995.

[3] Lian, S. and Cui, Y., 'Fractal and Its Application in Description of Spatial Information', Proceedings of the International Symposium on Digital Earth, Science Press, 1999.

[4] T. Kasparis, D. Charalampidis, M. Georgiopoulos, J. Rolland, Segmentation of textured images based on Fractals and image filtering, Pattern Recognition 34 (2001)1963-1973. 